INRA Prod. Anim.,

2013, 26 (4), 375-382

\title{
Regard philosophique sur la causalité en épidémiologie - approche interdisciplinaire
}

\author{
C. DUCROT ${ }^{1}$, D. BRES \\ ${ }^{1}$ INRA, UR 346 Epidémiologie animale, F-63122 Saint-Genès-Champanelle, France \\ ${ }^{2}$ Lycée Paul Eluard, 15-17 avenue Jean Moulin, F-93206 Saint-Denis, France \\ Courriel : christian.ducrot@clermont.inra.fr
}

Pour prétendre diminuer le risque de maladie en agissant sur certains facteurs, le préalable est de s'assurer que ces facteurs ont une relation causale avec la maladie. En illustrant les propos avec l'exemple de l'encéphalopathie spongiforme bovine, cet article interdisciplinaire aborde la question de la causalité en épidémiologie et l'apport de la philosophie sur ce sujet. Ces réflexions sont largement extrapolables aux disciplines d'observation.

L'épidémiologie s'intéresse pour l'essentiel à la prévention des maladies et, pour cela, aux enchaînements d'évènements qui se traduisent par (qui conduisent à) l'exposition de certains types d'individus à certains types d'environnement (MacMahon et Pugh 1970). Aussi, les épidémiologistes s'efforcent-ils d'identifier ces expositions et d'évaluer leur impact sur la santé. Une grande part de l'épidémiologie consiste à s'intéresser aux associations entre facteurs et maladie, associations qui, si elles sont de nature causale, permettent d'envisager des modes d'action destinés à améliorer la santé et le bien-être des individus et, dans le domaine vétérinaire, la productivité des animaux ainsi que la qualité et la sécurité sanitaire des aliments d'origine animale (Dohoo et al 2003). En généralisant le propos, d'autres disciplines de la biologie basées sur l'observation (en productions animales par exemple) ont aussi pour enjeu de comprendre l'effet de facteurs (nutritionnels, environnementaux, sociaux...) sur certains paramètres biologiques (croissance, production, qualité des produits...) à des fins de pilotage et d'amélioration de la production.

Ce qui rend difficile cette tâche, c'est l'extraordinaire intrication de facteurs de divers types, génétiques, individuels, environnementaux, sociaux, qui, par leurs associations complexes, concourent au risque de maladie. Ce concept de causes multiples en interaction trouve ses racines chez Hippocrate qui aborde vers 400 av. J-C, dans son traité des Airs, des Eaux et des Lieux, les habitudes de vie et les éléments environnementaux à analyser pour comprendre la santé des populations. Tombé dans l'oubli pendant des siècles, ce concept a progressivement refait surface à partir du XVIII ${ }^{\text {ème }}$ siècle et est revenu en force au cours des années 1970. Maintenant, les études épidémiologiques sur les facteurs impliqués dans les maladies intègrent très régulièrement des éléments à la fois sociaux, physiques et biologiques (Dohoo et al 2003). En parallèle, la méthodologie des études épidémiologiques évolue progressivement pour adapter les dispositifs d'étude et les méthodes d'analyse à cette complexité des situations étudiées.

L'objectif d'une étude épidémiologique analytique est la mise en évidence de relations statistiques significatives entre certains facteurs et une maladie donnée. Utiliser ces résultats en matière d'action pour prévenir et contrôler le risque de cette maladie, pose nécessairement la question cruciale de savoir si la relation mise en évidence est de nature causale ou non. Prenons à titre illustratif l'exemple de l'Encéphalopathie Spongiforme Bovine (ESB), à propos de laquelle les autorités sanitaires ont dû prendre des décisions majeures sur la base de résultats scientifiques incertains. En 1988 , deux ans après l'identification au Royaume-Uni de cette nouvelle maladie chez les bovins, les premières études épidémiologiques (Wilesmith et al 1988) ont montré une relation statistique entre la maladie et l'usage des farines animales dans l'alimentation des bovins. Vu la très longue incubation de la maladie (plusieurs années) et diverses difficultés méthodologiques, la confirmation expérimentale de la nature causale de la relation entre farines animales et ESB ne pouvait pas être obtenue avant longtemps. C'est sur la base de la relation épidémiologique observée, couplée à la nature histologique des lésions du cerveau qui rappelaient celles d'autres encéphalopathies spongiformes animales (tremblante du mouton) et humaines (maladie de Creutzfeldt-Jakob, kuru), dont l'une d'entre elles, le kuru, était connue pour être transmise par anthropophagie (Gajdusek 1977), que l'interdiction des farines animales dans l'alimentation des bovins a été mise en place au Royaume-Uni la même année ; elle s'est traduite par une décrue de l'épidémie observée six ans plus tard. La même maladie permet d'illustrer un débat polémique autour de la notion de causalité : quand huit ans plus tard, en 1996, a été annoncée par les autorités britanniques la découverte de cas humains d'un variant de la maladie de CreutzfeldtJakob, l'attribution de cette nouvelle maladie humaine à la consommation de produits d'origine bovine issus d'animaux atteints d'ESB a fait débat entre scientifiques et dans la société. Les éléments épidémiologiques en faveur de la relation causale entre les deux maladies étaient les circonstances temporelles et géographiques de l'arrivée des deux maladies, ainsi que quelques premiers éléments sur les habitudes alimentaires des personnes atteintes et une source potentielle commune pour certains des cas, en l'occurrence une boucherie. L'ESB a par ailleurs été transmise expérimentalement au singe, mais aucune expérimentation de certitude n'est bien entendu envisageable chez l'Homme. Sur la base des indices connus, les autorités britanniques et européennes ont adopté 
l'hypothèse de cette relation causale et mis en place des mesures de contrôle du risque pour l'Homme, notamment en supprimant de la consommation humaine les organes bovins à risque, notamment l'encéphale et la moelle épinière. Ces exemples montrent l'importance pour la gestion des risques de l'interprétation des résultats d'épidémiologie en terme de causalité, avec en corollaire des répercussions importantes, notamment en termes économiques et judiciaires. Au-delà de l'épidémiologie, le même questionnement existe dans toutes les disciplines travaillant sur des données d'observation, dispositifs dans lesquels il n'est pas possible de contrôler la présence de facteurs dont on veut étudier l'effet sur un phénomène biologique.

Quelques éléments techniques permettent à l'épidémiologiste de cerner au plus près la nature causale de la relation qu'il étudie, mais cette nature causale reste nécessairement du domaine de l'interprétation. Aussi l'objet de cet article estil d'apporter un éclairage philosophique aux épidémiologistes et plus largement aux biologistes sur cette question de la causalité, et ses conséquences possibles en termes de pratiques disciplinaires. Après avoir envisagé les outils de l'épidémiologiste pour approcher la notion de causalité à partir de ses travaux, nous aborderons plus longuement les apports de la philosophie à la question de la causalité, avant de discuter des apports possibles de ces réflexions à la pratique du biologiste ou de l'épidémiologiste.

\section{1 / Approche épidémiolo- gique}

\section{1 / Phénomènes sous-jacents à une relation statistique significa- tive}

En termes pragmatiques pour l'épidémiologiste, la mise en évidence d'une relation statistique significative entre un facteur et une maladie peut résulter de quatre mécanismes différents.

\section{a) Relation causale}

Le premier qui vient à l'esprit, et pour lequel on a conçu l'étude pour tester entre autres cette hypothèse biologique, est une relation de type causal entre le facteur et la maladie, la présence de celui-ci favorisant l'apparition de celle-là. L'importance de l'effet du facteur sur la maladie est mesurée traditionnellement par différents types d'estimateurs, les plus courants étant le risque relatif et le «odds ratio » (encadré 1, Dohoo et al 2003). L'interprétation de ces estimateurs repose sur le fait que plus ils ont des valeurs élevées au-dessus de la valeur

\section{Encadré 1. Le « Odds ratio ».}

En épidémiologie, la fréquence de cas de maladie parmi les individus exposés à un facteur donné est généralement comparée à la fréquence de la maladie chez ceux qui ne sont pas exposés à ce facteur, ce qui permet d'étudier l'association entre le facteur et la maladie.

En prenant l'exemple de l'ESB, dans une étude comparant des bovins atteints d'ESB à des animaux sains tirés au hasard pris comme témoins (Jarrige et al 2007), on peut comparer la proportion d'animaux atteints d'ESB parmi ceux ayant consommé un nombre plus ou moins important d'aliments du commerce différents entre la naissance et l'âge de deux ans (aucun, 1 à 3 , plus de 3 ).

Le fait qu'on sur-représente délibérément le nombre de cas d'ESB dans une étude de ce type (dite cas-témoins), afin de pouvoir étudier une maladie rare dans la population, augmente artificiellement les pourcentages de malades dans le graphique ci-dessous, par rapport à ce qui serait observé dans la population générale.

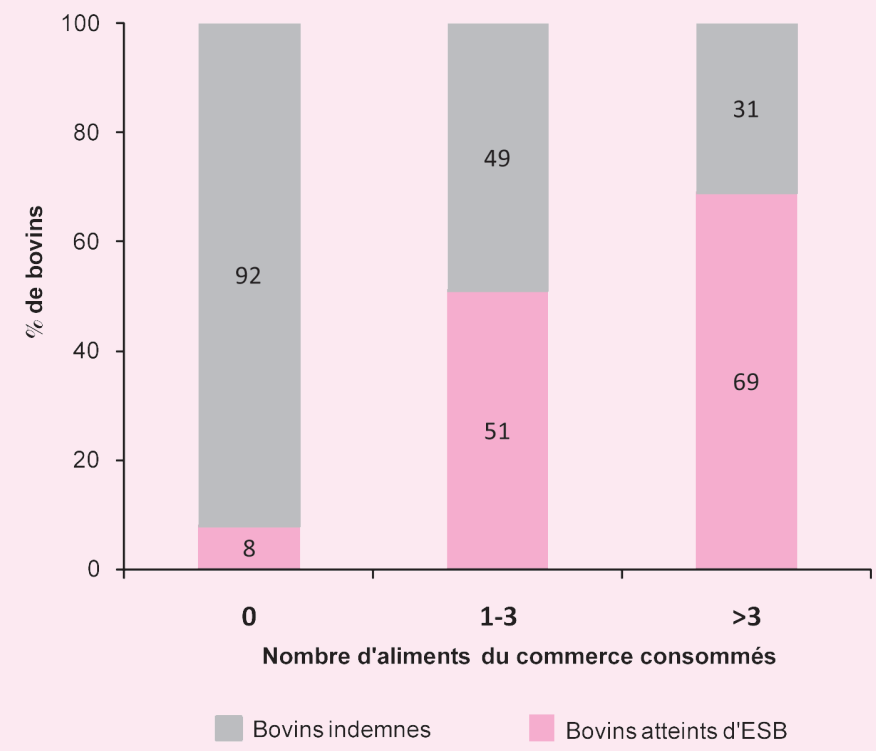

L'odds ratio (Dohoo et al 2003) permet de mesurer l'association observée. Dans l'exemple donné, on peut successivement comparer les bovins n'ayant consommé aucun aliment composé du commerce à ceux en ayant consommé 1 à 3 différents (calcul a), puis à ceux en ayant consommé plus de 3 (calcul b) :

a.

b. $\quad(0$ versus $>3)$ odds ratio $=(69 / 31) /(8 / 92)=25,6$

Le résultat montre que les bovins ayant consommé 1 à 3 types différents d'aliments composés du commerce dans le début de la vie ont eu un risque 12 fois supérieur (approximation de langage car ce n'est pas tout à fait cela) d'être atteints d'ESB comparé à ceux n'en ayant jamais consommé ; et 25 fois pour ceux en ayant consommé plus de 3 différents.

II est possible de calculer un intervalle de confiance du odds ratio. L'odds ratio est dit statistiquement significatif quand son intervalle de confiance, à un niveau donné de risque, n'inclut pas la valeur 1 (un odds ratio de 1 indique une absence totale d'association). Enfin, l'odds ratio est ajusté quand son calcul tient compte de la présence ou pas d'autres facteurs associés à la fois au risque de maladie et au facteur étudié.

seuil de 1 , plus leur présence augmente la probabilité que l'individu exposé au facteur soit malade, en comparaison à un individu non soumis au facteur. Il est à noter que même si la nature causale d'un facteur semble avérée dans un contexte donné, cela ne signifie pas néanmoins que cet effet est vrai et constant dans toutes circonstances et toutes populations. A titre illustratif, l'exposition alimentaire des bovins aux farines animales au Royaume-Uni à la fin des années 1980 était la cause de l'ESB (Ducrot et al 2004, 2008). Néanmoins, dans un autre contexte, par exemple avec un degré de stérilisation beaucoup plus poussé des farines animales avant leur utilisation alimentaire, on peut faire l'hypothèse que l'exposition alimentaire aux farines animales induirait un risque moindre, car ces farines animales seraient porteuses de moins d'infectiosité ; on a d'ailleurs utilisé des farines animales dans l'alimentation des bovins depuis les années 1930 et dans de nombreux pays sans manifestation visible de maladie jusqu'aux années 1980 au Royaume-Uni.

\section{b) Hasard}

Le deuxième mécanisme expliquant une relation statistique significative entre 
un facteur et une maladie est le hasard. En effet, quand on affirme par exemple que telle relation est statistiquement significative au seuil de $5 \%$, cela sous-entend que si cette relation était en réalité inexistante à l'échelle de la population générale, on serait néanmoins susceptible de l'observer dans $5 \%$ des échantillons d'individus tirés au hasard dans cette population, ceci découlant des aléas de l'échantillonnage. On réduit ce risque d'observer par hasard une relation statistiquement significative en diminuant le seuil de risque qu'on accepte, à $1 \%$ ou $1 \%$ par exemple, mais surtout en limitant le nombre de facteurs étudiés dans le cadre d'une étude épidémiologique et en les focalisant sur des hypothèses causales fortes. Par ailleurs, quand c'est envisageable, observer une relation dose-effet, avec un niveau de risque de maladie augmentant en parallèle avec l'intensité du facteur de risque, ne plaide pas en faveur d'une relation due au hasard.

\section{c) Facteur de confusion}

Le troisième mécanisme possible est la présence d'un facteur de confusion, qui crée artificiellement une relation statistique entre un facteur et une maladie, si ce facteur est statistiquement corrélé à la fois au facteur étudié et à la maladie. Prenons un exemple pour l'illustrer. On sait que l'ESB est plus fréquente chez les vaches laitières que chez les vaches allaitantes. Imaginons une région fortement peuplée en vaches laitières et une autre en vaches allaitantes. Analyser le risque ESB en fonction de la région seulement pourrait faire penser qu'une zone géographique donnée (celle avec une forte densité de vaches laitières) est plus à risque, ce qui amènerait peut-être à se poser des questions sur les pratiques des fabricants d'aliments dans cette région, alors qu'elle est seulement peuplée de vaches laitières qui sont elles-mêmes plus à risque. Pour déjouer ce type de confusion, seule une bonne connaissance du terrain, des pratiques d'élevage, et des habitudes peut permettre d'imaginer les facteurs de confusion possibles afin de les intégrer dans l'analyse. En effet, les outils statistiques permettent de résoudre ce type de problème dès lors qu'on l'a identifié et qu'on a pris les moyens de collecter l'information sur les facteurs de confusion.

\section{d) Indicateur de risque}

Le quatrième et dernier mécanisme possible expliquant une relation statistique est le fait que le facteur lié statistiquement à la maladie n'est pas en luimême un élément causal de la maladie, mais un indicateur de la présence d'autres facteurs eux-mêmes causes de la maladie. Ceci est rencontré particulièrement dans des systèmes très structurés, dans lesquels de nombreux paramètres sont interdépendants, ce qui est très souvent le cas en épidémiologie. Par exemple, en matière d'élevage bovin laitier, les troupeaux à très forte production laitière utilisent de manière préférentielle certaines races de vaches, planifient la première mise bas des génisses à un jeune âge, et ont une forte intensification alimentaire à la fois dans le jeune âge (pour permettre d'atteindre le poids adulte rapidement) et pendant la lactation (pour permettre un niveau de production laitière élevé). Identifier par exemple comme facteur de risque d'ESB une production laitière très élevée ( $\mathrm{La}$ Bonnardière et al 2007) pose la question des facteurs causaux sous-jacents : la race, l'alimentation des jeunes, l'alimentation des vaches adultes, ou la forte production elle-même? Décortiquer ces facteurs est souvent difficile, mais nécessite a minima de bien connaître les pratiques de terrain, les habitudes et les systèmes d'élevage.

\section{2 / Approche de la causalité en épidémiologie}

Pour tenir compte de ces chaussetrappes, l'épidémiologie a mis en place au fil du temps des pratiques et des méthodes à même d'en limiter les risques.

\section{a) Conception de l'étude et analyse sta- tistique}

Un des éléments forts de ces pratiques est de réfléchir et concevoir en amont de l'étude un schéma des hypothèses, ou diagramme des causes (Dohoo et al 2003), à savoir un schéma synthétisant ce qu'on sait des relations entre facteurs clés et ce qu'on fait comme hypothèses fortes expliquant le risque de maladie. Un tel schéma (encadré 2) est d'autant plus riche et proche de la réalité qu'on connaît bien le terrain sur lequel on travaille, à la fois la population et la maladie étudiées. Un tel schéma permet à la fois d'amener le chercheur à focaliser son étude sur un nombre limité de facteurs, ce qui permet de limiter le risque de relations statistiques dues au hasard, mais aussi d'identifier les possibles facteurs de confusion afin de les prendre en compte dans l'étude et son analyse. Une autre pratique est de s'entourer de personnes connaissant bien le milieu d'étude, par exemple le monde de l'élevage dans le domaine animal, car leur expertise est

\section{Encadré 2. Le schéma des hypothèses.}

Le schéma des hypothèses (Dohoo et al 2003) simplifié et non exhaustif sur le risque ESB présenté ci-dessous illustre l'enchaînement des effets relatifs à l'alimentation des jeunes et des adultes sur le risque d'infection par l'ESB dans les années 1990 (La Bonnardière et al 2007, Jarrige et al 2007, Ducrot et al 2008). II aborde la question au niveau de l'exploitation agricole et est bâti sur l'hypothèse que la contamination par l'ESB est liée aux aliments du commerce (il y avait dans les années 1990 présence d'infectiosité dans les farines animales et possibilité de contaminations croisées entre aliments pour animaux monogastriques (porcs, volailles), dans lesquels les farines animales étaient alors autorisées, et aliments pour bovins (Ducrot et al 2008)

Dans ce schéma qui distingue les facteurs agissant pendant la période de croissance et ceux agissant à l'âge adulte, pendant la période de production (laitière notamment), on voit que l'action de certains facteurs sur le risque n'existe que combinée à d'autres facteurs, ce qui renvoie à des phénomènes d'interaction à prendre en compte lors de l'analyse ; à titre d'exemple, la quantité de concentrés nécessaire combinée avec la proportion d'aliments achetés dans le commerce agissent sur le risque d'infection. Par ailleurs, d'autres facteurs ont un effet sur le risque par une cascade d'effets, par exemple le rôle du mois de naissance.

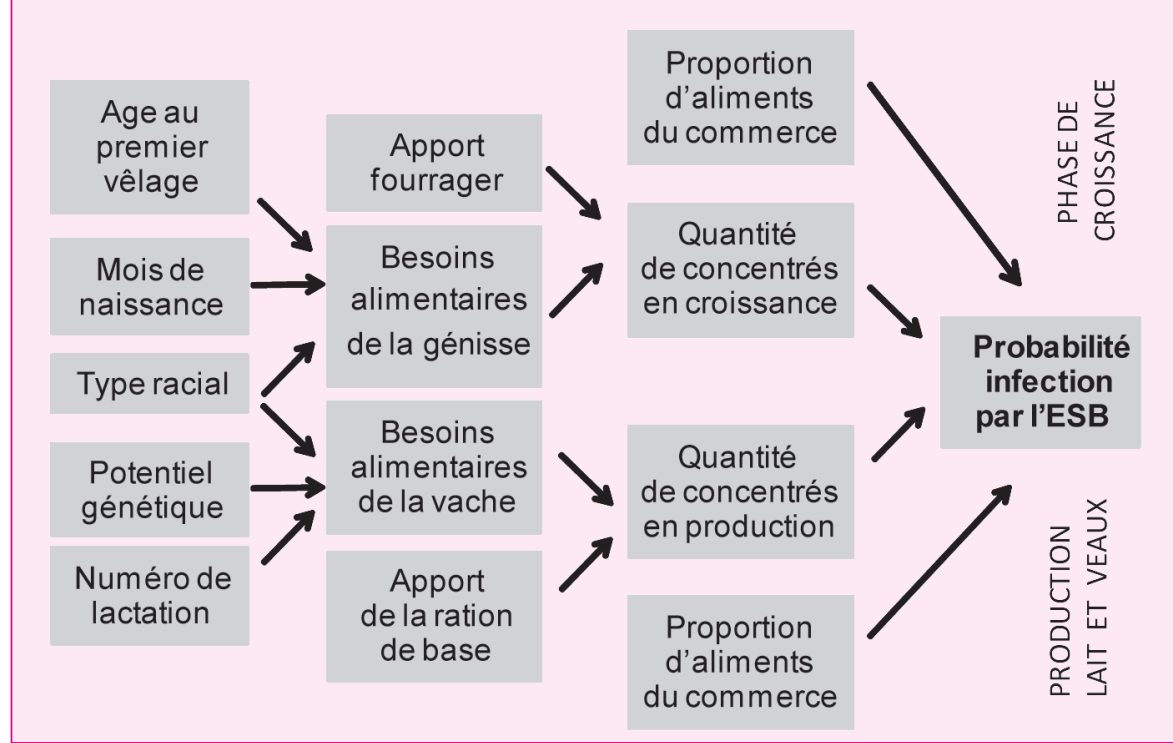




\section{Encadré 3. Les critères de Hill.}

L'exemple pris pour présenter l'odds ratio (cf. encadré 1) permet d'illustrer certains des critères de Hill (1965). Cet exemple montre l'effet du nombre d'aliments du commerce différents consommés entre la naissance et deux ans sur le risque d'infection par l'ESB.

Il y a bien séquence dans le temps entre le facteur et son effet : il a été spécifiquement enquêté sur le mode d'alimentation en début de vie de l'animal considéré (cas d'ESB ou témoin), période a priori la plus à risque d'infection, pour étudier son effet sur le risque d'atteinte par l'ESB observée des années plus tard.

La force de l'association observée est grande, avec des odds ratios très élevés (ainsi que leur intervalle de confiance, non montré dans cet exemple simplifié).

La notion de dose-réponse est aussi présente dans la mesure où plus le nombre d'aliments du commerce différents est élevé, plus le odds ratio augmente (odds ratio de 12 pour 1 à 3 aliments différents, de 25 pour plus de 3 aliments différents).

Enfin, la relation est plausible et cohérente avec les hypothèses de présence d'infectiosité dans les farines animales et de contaminations croisées entre aliments pour animaux monogastriques dans lesquels les farines animales étaient autorisées à l'époque et aliments pour bovins. Si c'est le cas, on doit s'attendre en effet à ce que les animaux consommant des aliments du commerce dans le jeune âge soient plus à risque, et que plus ils consomment d'aliments différents du commerce, plus ils aient une probabilité augmentée d'avoir consommé un aliment contaminé.

En revanche, dans cet exemple, la preuve expérimentale serait extrêmement difficile à apporter, ne serait-ce que parce qu'une expérimentation devrait durer a minima plus de 4 ans en milieu contrôlé, compte tenu de la longue durée d'incubation de l'ESB.

précieuse depuis la conception de l'étude jusqu'à l'interprétation des résultats obtenus, notamment pour analyser si les facteurs mis en évidence risquent d'être les indicateurs d'autres facteurs euxmêmes causaux.

\section{b) Interprétation}

Quand toutes ces étapes permettant de limiter le risque de relations statistiques non causales ont été franchies, reste néanmoins la phase finale d'interprétation des résultats en terme de causalité, avec toujours le doute et la question en suspens de savoir si notre méconnaissance du problème a pu nous faire oublier un élément clé. Les épidémiologistes se sont dotés à ce stade d'un ensemble de « critères de causalité » décrits par Hill (1965) et permettant de discuter les résultats épidémiologiques, avec l'idée que plus le nombre de critères vérifiés est important, plus la relation causale est probable ; ils abordent entre autres la séquence dans le temps entre facteur et maladie, la force de l'association observée, ainsi que les notions de dose-réponse, plausibilité, cohérence, spécificité, analogie et preuve expérimentale (encadré 3). Ces critères sont néanmoins en partie critiqués dans l'ouvrage de Dohoo et al (2003), car ils risquent parfois d'amener à rejeter des explications causales valides si on les considère avec trop de rigueur; ils ne laissent pas place à des connaissances nouvelles inattendues.

\section{2 / Regard philosophique}

L'interprétation des résultats en termes de causalité reste délicate d'autant plus qu'elle n'est pas sans poser un certain nombre de problèmes au niveau philosophique.

\section{1 / Cause efficiente et cause finale}

La causalité est un thème de réflexion qui a fait l'objet d'importants développements philosophiques dès lors que le savoir théorique était défini par Aristote comme connaissance des causes ${ }^{1}$ (Aristote, édition 1979). Aristote est aussi l'auteur d'une distinction entre cause efficiente et cause finale qui sera largement reprise. Elle permettra à la science moderne, née de la révolution galiléenne, de circonscrire son domaine à la recherche des causes efficientes, la cause « efficiente » ou « motrice » renvoyant à la cause productrice d'une chose tandis que la cause « finale » définirait sa raison d'être, ce en vue de quoi la chose existe (Aristote, édition 1931). On peut considérer qu'à partir du XVII ${ }^{\text {ème }}$ siècle, la science abandonne la recherche des causes finales pour se limiter à la recherche des causes efficientes. Il est admis de façon plus ou moins explicite que le scientifique se contente de montrer comment les phénomènes naturels s'enchaînent sans chercher leur raison d'être dans quel but ils se produisent. Cet usage plus rigoureux de la notion de causalité participe à la constitution de la science.

Cependant, cette notion demeure encore problématique pour la philosophie qui cherche à mieux la définir afin de déterminer jusqu'à quel point son usage est pertinent dans les sciences. Ainsi, la réflexion philosophique sur la science déjà constituée part de la constatation suivante : les répétitions et régularités observées dans la nature (Carnap 1973) conduisent le scientifique à la formulation de lois qui sont souvent interprétées comme traduisant des relations de causalité entre les phénomènes naturels. Mais que signifie exactement l'idée qu'un événement serait la cause d'un autre? Cette idée n'est évidente qu'en apparence. D'un côté, le " principe de causalité » encore appelé « principe de raison suffisante » est au fondement de la rationalité scientifique : " rien n'est sans raison ${ }^{3}$ (Leibnitz, édition 1969). Pour la science, ce principe ne signifie pas que tout ce qui arrive est justifié à exister mais seulement que tout phénomène a une cause : en théorie, tout est explicable rationnellement. La notion de raison n'est pas à comprendre ici au sens de justification, mais au sens de cause. Par ailleurs, la notion de causalité est encombrée de présupposés métaphysiques ${ }^{4}$ qu'il convient d'identifier.

\section{2 / Réalité absolue et perception de la réalité}

On peut schématiquement identifier trois niveaux de problèmes dans l'usage de la notion de causalité. Le premier problème se situe à un niveau très général. L'attitude naturelle considère l' « explication » scientifique des phénomènes naturels comme une connaissance de la nature des choses. Elle ne distingue pas la réalité absolue, telle qu'elle existe indépendamment de l'esprit connais-

\footnotetext{
1 « Nous estimons posséder la science d'une chose d'une manière absolue... quand nous croyons que nous connaissons la cause par laquelle la chose est, que nous savons que cette cause est celle de la chose, et qu'en outre il n'est pas possible que la chose soit autre qu'elle $n$ 'est ».

2 «Dans une troisième acception, la cause est le principe premier d'où vient le mouvement ou le repos... ce qui produit le changement est cause du changement produit... En dernier lieu, la cause signifie la fin, le but et c'est alors le pourquoi de la chose ». La cause motrice ou efficiente est définie comme « le principe d'où part le mouvement», la cause finale comme ce en vue du quoi la chose existe : "Ainsi la santé est la cause de la promenade ».

3 « Jamais rien n'arrive, sans qu'il y ait une cause ou du moins une raison déterminante, c'est-à-dire quelque chose qui puisse servir à rendre raison... pourquoi cela est existant plutôt que non existant et pourquoi cela est ainsi plutôt que de toute autre façon ».

4 Par métaphysique, il faut entendre ici un domaine de questions qui concernent la connaissance de la réalité absolue et ne peuvent recevoir une réponse scientifique.
} 


\section{Encadré 4. Kant et la critique de la raison pure.}

Emmanuel Kant, philosophe allemand du XVIII ${ }^{2}$ è siècle, a montré la nécessité pour la raison de se livrer à un examen de son pouvoir de connaître dans La Critique de la Raison pure. Cet examen a pour résultat de limiter ses prétentions à la seule connaissance des " phénomènes ». Par " phénomènes », il faut entendre les choses telles qu'elles apparaissent au sujet humain qui ne peut être certain de l'existence de quoi que ce soit, tant qu'il n'en fait pas une expérience qui passe d'abord par ses sens. Le sujet humain ne peut connaître ce que sont les choses en elles-mêmes indépendamment de la façon dont il les perçoit. Cela ne signifie pas que chacun en reste à une expérience privée, subjective des choses, mais seulement que la connaissance que l'Homme en général peut avoir de la réalité reste nécessairement limitée par sa " vision » humaine des choses. Les formes de ses facultés de connaître, "sensibilité » ou capacité de recevoir des impressions sensibles et " entendement » ou faculté de former des concepts, déterminent la façon dont il peut accéder à la réalité. Kant prétend avoir opéré une " révolution copernicienne » dans la réflexion philosophique sur la connaissance. Ce sont les objets qui doivent se régler sur notre faculté de connaître et non l'inverse.

sant ${ }^{5}$ et la réalité telle qu'elle peut apparaître à l'Homme, étant donné ses facultés de connaissance. Elle confond ce que le philosophe distingue : les « choses en soi » et les «phénomènes » (encadré 4). De ce point de vue, la causalité est un « concept à double face » (Yakira 1993). On peut la considérer comme un élément fondamental de la réalité objective, existant en soi indépendamment de l'esprit connaissant, ou bien seulement comme un concept déterminant les modalités de la rationalité scientifique : une chose est rationnellement connue quand on en connaît sa (ses) cause(s). La philosophie kantienne conduit à distinguer ces deux possibilités et affirme la finitude de notre pouvoir de connaître : nous ne pouvons pas connaître les choses telles qu'elles seraient en soi, indépendamment de la forme que notre esprit leur prête spontanément afin de les connaître. La causalité est un « concept a priori » de l'entendement qui impose ses formes aux flux des impressions reçues par les sens afin que la réalité devienne objet de connaissance (Kant, édition 1990). Dans cette perspective, le scientifique tout comme l'Homme du commun n'appréhendent qu'une réalité déjà modifiée par leurs facultés de connaissance. Il n'y a pas de connaissance possible de la réalité absolue. On ne peut faire à son sujet que des spéculations qui ne peuvent être vérifiées par aucune expérience. En effet, selon l'analyse kantienne, il y a des conditions de possibilité de l'expérience qui consistent dans la mise en forme spontanée des données des sens par l'esprit humain. «Il se pourrait bien que... notre connaissance par expérience fût un composé de ce que nous recevons des impressions sensibles et de ce que notre propre pouvoir de connaître (simplement excité par des impressions sensibles) produit de lui-même » (Kant, édition 1990). Toute expérience de la réalité supposant sa mise en forme par l'esprit, il n'y a donc aucune expérience possible de la réalité absolue. Au vu de cette analyse, nous ne pouvons exclure que la causalité ne soit pas dans les choses, mais n'exprime que la manière dont l'être humain perçoit nécessairement les choses. Une question demeure sans réponse certaine : le «principe de causalité » révèle-t-il un caractère interne de la réalité objective ou seulement la structure de notre esprit qui ne peut concevoir d'événement sans cause ? Cette question n'a pas nécessairement de conséquences pratiques sur le travail du scientifique ( si ce n'est de l'inviter à une certaine modestie).

\section{3 / Conjonction constante et connexion nécessaire}

Le second problème concerne moins l'existence objective de la relation de causalité que sa définition et la difficulté

\section{Encadré 5. Auguste Comte et le positivisme.} humaine. d'identifier des causes à partir de l'observation des phénomènes naturels. Prenant acte des difficultés liées à la notion de cause, le «positivisme » fondé au XIX ${ }^{\text {ème }}$ siècle par Auguste Comte (encadré 5) a voulu substituer la recherche des lois à la recherche des causes. En effet, au XVIII ${ }^{\text {ème }}$ siècle, le philosophe Hume (Hume, édition 1983) avait soumis la notion de cause à une critique dévastatrice. Cette notion connote intuitivement une action de « force», une " production » et une « connexion nécessaire ». Cependant, ces trois notions ne représentent rien qui existe de façon certaine dans les choses selon le critère empiriste du savoir. Tout d'abord, il n'existe aucune perception possible d'une « connexion nécessaire » entre deux phénomènes. Ainsi l'expérience nous révèle-t-elle des liaisons constantes mais non des liaisons nécessaires. Le passage de l'idée de « conjonction constante " à celle de « connexion nécessaire » est sans fondement logique. C'est seulement l'habitude qui porte l'esprit à croire que le futur sera conforme au passé selon la célèbre analyse de Hume. Par ailleurs, liaison constante entre deux phénomènes ne signifie pas nécessairement relation de succession. Ainsi peut-on constater l'existence d'une constante entre le volume d'un gaz et sa pression (loi de Boyle) sans que l'un puisse être considéré comme la cause de l'autre. C'est pourquoi selon le fondateur du positivisme Auguste Comte (Comte, édition 1975), la science ne doit pas avoir pour ambition de chercher les causes des phénomènes, mais doit se contenter de montrer comment ces phénomènes s'enchaînent, c'est-à-dire selon quelles lois. Les « lois » sont des

Dans sa version initiale, celle d'Auguste Comte, le " positivisme » est une philosophie qui lit dans l'histoire un progrès de la raison et distingue différentes étapes dans « la marche générale de l'esprit humain ». Le développement de l'intelligence humaine serait assujetti à une loi fondamentale : la " loi des trois états ». Le premier âge de l'humanité ou l'« état théologique » est caractérisé par une prédilection pour les questions insolubles. "L'esprit humain, dirigeant essentiellement ses recherches vers la nature intime des êtres, les causes premières et finales, en un mot, vers les connaissances absolues, se représente les phénomènes comme produits par l'action directe et continue d'agents surnaturels, dont l'intervention arbitraire explique toutes les anomalies de l'univers » (Comte, édition 1975). L'« état métaphysique » ou l'adolescence de l'humanité substitue aux divinités surnaturelles des entités abstraites pour expliquer les phénomènes naturels, par exemple, le principe vital en biologie. L' " état positif » ou " scientifique » qui est le régime définitif de la raison humaine, se caractérise par l'intérêt exclusif pour les faits et le rejet des spéculations métaphysiques. "L'esprit humain reconnaissant l'impossibilité d'obtenir des notions absolues, renonce à chercher l'origine et la destination de l'univers, et à connaître les causes intimes des phénomènes, pour s'attacher uniquement à découvrir, par l'usage bien combiné du raisonnement et de l'observation, leurs lois effectives, c'est-à-dire leurs relations invariables de succession et de similitude » (Comte, édition 1975). "Positif » désigne le réel par opposition au chimérique, l'utile par opposition à l'oiseux, la certitude par rapport à l'indécision, le précis par opposition au vague. Avec l'idée de « loi des phénomènes ", la science découvre à la fois le fondement et les limites de la connaissance 
《 relations fonctionnelles » entre les phénomènes. Elles n'impliquent pas de penser les phénomènes mis en relation comme si l'un était la cause productrice de l'autre. Le « principe du déterminisme » affirme seulement que tous les phénomènes sont liés les uns aux autres par des relations invariables appelées « lois » sans prétendre mettre en évidence des causes. L'explication scientifique consiste seulement à montrer qu'un certain nombre de conditions sont suffisantes pour produire un phénomène. Cela n'autorise pas à conclure que ces conditions sont les seules capables de le produire, qu'elles sont « les » causes du phénomène en question. Dans une perspective positiviste, les « lois causales » n'expriment qu'une corrélation entre deux événements et non un acte de production interne d'un phénomène par un autre.

\section{4 / Problème de l'induction}

Le troisième problème constitue une reprise de la critique humienne (Hume, édition 1983) de la causalité, reformulée par l'épistémologie au XX ${ }^{\text {ème }}$ siècle sous l'expression de «problème de l'induction ». Par induction, on entend le raisonnement par lequel on tire une règle générale à partir de l'observation d'une liaison constante entre deux phénomènes (appelé inférence causale en épidémiologie). Aussi grand soit le nombre d'expériences venant étayer une hypothèse causale, celle-ci ne peut être prouvée d'une manière définitive. «Tous les exemples qui confirment une vérité générale ne suffisent pas pour établir la nécessité universelle de cette même vérité ") notait déjà Leibnitz (Leibnitz, édition 1990) dans Les Nouveaux Essais. Quel que soit le domaine scientifique envisagé, il n'est jamais légitime d'inférer des lois universelles à partir d'observations particulières. Plusieurs raisons peuvent être invoquées. En premier lieu et d'un point de vue théorique, il ne suit pas nécessairement que ce qui est arrivé arrivera toujours de même. Rien ne nous assure avec une absolue certitude de la constance de la nature. Une certaine indétermination semble à l'œuvre. Le «principe du déterminisme », selon lequel les mêmes causes produisent les mêmes effets dans les mêmes conditions, n'est qu'un postulat méthodologique. La science part du principe que les phénomènes naturels n'arrivent pas de manière aléatoire, mais s'enchaînent selon des lois rendant possible leur prévision. Mais elle n'a pas la preuve absolue qu'il en est toujours ainsi. En second lieu et d'un point de vue pratique, le scientifique n'est jamais certain d'avoir repéré et isolé tous les facteurs qui concourent à l'apparition d'un phénomène. Ceci a été amplement développé dans la première partie de notre article.

\section{5 / Néopositivistes et falsifica- tionistes}

Il est donc impossible de «vérifier» de façon définitive la validité d'une hypothèse causale. Mais «si dans un grand nombre d'exemples, une chose d'un certain genre est associée d'une certaine manière à une chose d'un autre genre, il est probable qu'une chose du premier genre soit toujours pareillement associée à une autre chose de l'autre genre, et à mesure que croit le nombre d'exemples, la probabilité se rapproche indéfiniment de la certitude " selon Bertrand Russell (Russel, édition 2002). Ainsi les philosophes s'inspirant du «néo-positivisme » ou « empirisme logique » comme Rudolf Carnap préconisent-ils de chercher à « confirmer » l'hypothèse causale par l'apport de nouveaux résultats favorables. Développant cette idée, C. Hempel (Hempel 1972) énonce que la conjonction d'arguments différents et complémentaires est essentielle pour donner du poids à la nature causale des résultats. Afin d'augmenter le degré de probabilité de cette hypothèse, les « falsificationistes » comme Karl Popper (Popper 1973) proposent, au contraire, de pousser l'hypothèse dans ses retranchements en la soumettant de façon systématique à de nouveaux tests visant à la réfuter. Il s'agit de tester l'inverse des conséquences logiques d'une hypothèse.

\section{3 / Implications de ces réflex- ions en biologie}

\section{1 / Notion de causalité}

L'examen des difficultés philosophiques liées à l'usage de la notion de causalité ne conduit pas pour autant à y renoncer. Certes, le principe de causalité au sens du principe de raison suffisante est indémontrable, c'est un postulat. Cependant, toute science véritable part du principe que les faits n'arrivent pas par hasard : leur production est la conséquence de faits qui les ont précédés. Ce principe est fécond puisqu'il permet à la connaissance scientifique de progresser. On peut alors considérer que la notion de causalité peut être conservée dans les sciences de la nature à la condition suivante : considérer qu'un énoncé sur une relation de causalité décrit une régularité observée dans la nature et rien de plus ${ }^{6}$ (Carnap, édition 1973). On peut toujours s'interroger philosophiquement sur l'existence de relations causales dans la réalité absolue comme nous l'avons indiqué dans notre premier point. Mais force est de reconnaître qu'intuitivement il est difficile de se passer de la notion de causalité pour se représenter l'enchaînement des phénomènes naturels. Cependant, il ne s'agit là que d'une limite, peutêtre provisoire, de la pensée non scientifique que le scientifique est invité à dépasser.

Une fois admise ces réserves, la question se pose alors de savoir jusqu'à quel point il est possible d'être certain d'une relation causale ou, du moins, d'une association constante entre deux phénomènes. Sur ce point, il apparaît clairement qu'aucune confirmation définitive n'est envisageable. On augmente la probabilité d'une hypothèse causale en variant les expérimentations susceptibles de la confirmer et en cherchant à la disqualifier afin de tester sa résistance (Popper 1973), sachant la difficulté de tester une hypothèse isolément. En effet, lorsqu'une observation semble infirmer une hypothèse, il reste difficile de savoir si c'est bien l'hypothèse qui est réfutée ou des hypothèses auxiliaires. Toutes ces considérations ne peuvent inviter qu'à la plus grande vigilance dans l'exercice de la science.

\section{2 / Déclinaisons pratiques en épidémiologie et dans d'autres disciplines}

Ces apports philosophiques ont plusieurs déclinaisons dans la pratique de l'épidémiologie. En premier lieu, l'épidémiologie n'est pas la seule discipline biologique à se poser la question de l'interprétation des résultats d'études en termes de causalité, même si cette question est un peu moins prégnante pour des approches expérimentales dans lesquelles de nombreux facteurs sont contrôlés ${ }^{7}$. Comme cela est bien souligné par la philosophie, il est impossible de vérifier de façon définitive la validité d'une hypothèse causale. Aussi, si la qualité du dispositif d'étude est déterminante dans le degré de confiance qu'on peut avoir dans la nature causale des relations mises en évidence, la discussion des résultats est aussi un acte majeur, d'autant plus important en épidémiologie et dans les disciplines d'observation dans lesquelles on ne peut pas fixer et contrôler comme on le souhaite les effets de facteurs confondants.

\footnotetext{
6 «Un énoncé sur une relation de causalité est un énoncé conditionnel. Il décrit une régularité observée dans la nature, et rien de plus ».

${ }^{7} \mathrm{Il}$ y a un continuum, de l'observation pure à des dispositifs expérimentaux extrêmement contrôlés, en passant par l'épidémiologie, basée sur une observation planifiée, et des dispositifs expérimentaux dans lesquels peu de facteurs sont contrôlés.
} 
a) Réfutation, champ d'application $d u$ résultat obtenu

Dans cet esprit, l'approche de Karl Popper est extrêmement intéressante car elle conduit à mieux questionner le résultat obtenu et à rechercher les contextes qui seraient le plus à même de le remettre en question. En arrière plan, cette approche par la réfutation a pour autre intérêt d'analyser dans quelles limites et dans quel champ d'application le résultat obtenu est valide en termes de causalité. A titre d'illustration, mettre en évidence dans certains pays uniquement une relation statistique entre consommation de farines animales par les vaches et apparition de cas d'ESB aurait du sens s'il s'avère que les autres pays n'ont pas importé de farines animales contaminées des pays infectés, et ont maintenu par ailleurs un dispositif de traitement et un degré de stérilisation des farines animales qui garantissent leur innocuité. La réflexion de Popper amène aussi à garder néanmoins à l'esprit qu'infirmer une hypothèse à partir d'une expérience donnée n'amène pas à infirmer systématiquement l'hypothèse, dans la mesure où sa non vérification dans ce contexte là peut n'être que conjoncturelle, due à l'intervention d'un autre élément du système.

\section{b) Conjonction d'arguments différents et complémentaires}

Un autre apport philosophique important est celui de Hempel (1972), selon lequel la conjonction d'arguments différents et complémentaires est essentielle pour donner du poids à la nature causale des résultats. Cette approche renvoie aux dix critères préconisés par Hill (1965) pour discuter la nature causale d'une relation statistique, la plausibilité de cette relation causale étant d'autant plus forte qu'un plus grand nombre de critères sont vérifiés. D'autres recommandations peuvent être proposées à l'épidémiologiste, qui découlent de cette pensée : i) aborder une question épidémiologique par différentes approches complémentaires, par exemple associant des approches à l'échelle de l'individu (par exemple étude comparant l'exposition de
« cas » de maladie à celle de « témoins » non atteints) à d'autres études menées à l'échelle géographique (par exemple études spatiales), ii) conforter les résultats épidémiologiques par une approche d'une autre discipline qui va décortiquer et faire en sorte d'analyser les ressorts à même d'expliquer les relations observées, par exemple une approche en sciences sociales complémentaire de l'approche épidémiologique). Ces deux façons de procéder ont été appliquées aux travaux menés pour comprendre les sources de contamination des vaches par l'agent de l'ESB, alors même que les farines animales avaient été interdites et qu'on observait encore des animaux atteints bien que nés après l'interdiction : associations d'études à l'échelle de l'individu et à différentes échelles géographiques (Jarrige et al 2007, Ducrot et al 2008), approche complémentaire en sciences sociales (Ducrot et al 2013). Dans le même esprit, une approche complémentaire peut aussi reposer sur une étude expérimentale visant à reproduire l'effet d'un facteur identifié, quand cette expérimentation est possible, ainsi que la modélisation ou d'autres études visant à expliciter le mécanisme biologique de facteurs identifiés.

\section{c) Regard critique sur les résultats}

Enfin, les différents problèmes relevés et analysés par le philosophe sur l'usage de la notion de causalité devraient amener l'épidémiologiste, et plus généralement le biologiste, à beaucoup d'humilité face à ses résultats. S'il est une idée clé à retenir de ce regard philosophique, c'est d'avoir un regard critique sévère sur son résultat, seule façon d'ajouter du poids et de la probabilité à son interprétation en terme de causalité. En ce qui concerne la recherche théorique de la vérité, Descartes considère que le doute radical est nécessaire. On ne doit tenir pour vrai que ce qui est absolument indubitable. Cependant, depuis l'époque de Descartes, la science a dû faire le deuil de l'exigence cartésienne d'une certitude absolue. Par ailleurs, même Descartes convient que dans la pratique, lorsqu'il s'agit d'agir et qu'il est néces- saire de prendre une décision, une autre approche doit être adoptée. Dans son discours de la méthode (Descartes, édition 2000) il écrit « afin que je ne demeurasse point irrésolu en mes actions, pendant que la raison $m$ 'obligerait de l'être en mes jugements, ... je me formai une morale par provision, qui ne consistait qu'en trois ou quatre maximes $»$. La seconde maxime aborde le fait que «les actions de la vie ne souffrant souvent aucun délai, c'est une vérité très certaine que, lorsqu'il n'est pas en notre pouvoir de discerner les plus vraies opinions, nous devons suivre les plus probables ». En d'autres termes, on est bien obligé d'accepter le probable comme certain pour prendre des décisions. Agir (pour supprimer une cause) est aussi un moyen a posteriori de conforter l'hypothèse causale (considérée comme vraie), en cela que cette action permet de tester l'effet de la suppression de la cause ; on s'attend à voir décroitre aussi la conséquence. Pour reboucler sur l'exemple de l'ESB, les mesures successives prises pour contrôler le risque de transmission entre vaches, sur la base - pour l'essentiel - de résultats épidémiologiques, a permis de juguler l'épidémie en une dizaine d'années, et de voir après coup une concordance entre la mise en place de mesures de contrôle successives de plus en plus drastiques et le niveau de décroissance du taux d'infection des animaux (Ducrot et al 2010).

\section{Conclusion}

La réflexion philosophique conforte l'idée que la nature causale d'une relation biologique statistiquement significative relève de l'interprétation et ne peut être prouvée dans l'absolu. Ceci est particulièrement vrai dans les disciplines d'observation, au nombre desquelles l'épidémiologie, mais également en expérimentation. Elle donne, par ailleurs, quelques pistes de réflexion dans la façon de conduire la recherche pour conforter le plus possible l'interprétation quant à la causalité d'une relation observée. 


\section{Références}

Aristote, édition 1931. Physique, II, III. Les belles lettres ed., Paris, France,192p.

Aristote, édition 1979. Seconds analytiques, I, 2 Vrin ed., Paris, 251p.

Carnap R., édition 1973. Les fondements philosophiques de la physique. Armand Colin Editions, Paris, France, 285p.

Comte A., édition 1975. Cours de philosophie positive. Hermann Editions, Paris, France.

Descartes R., édition 2000. Discours de la méthode. Le livre de poche Editions, Paris, $254 \mathrm{p}$.

Dohoo I., Martin W., Stryhn H., 2003. Veterinary epidemiologic research. Atlantic Veterinary College, Canada, Charlottetown, $706 \mathrm{p}$.

Ducrot C., Cabaret J., Touzeau S., Abrial D., Jacob C., Quiquampoix H., Grosclaude J., Gruner L., 2004. Épidémiologie de la tremblante et de l'Encéphalopathie Spongiforme Bovine en France. In: Numéro hors série, Encéphalopathies spongiformes transmissibles, Ducrot C., Charley-Poulain J., Aynaud J.M. (Eds). INRA Prod. Anim., 17, 67-76.

Ducrot C., Arnold M., de Koeijer A., Heim D., Calavas D., 2008. Review on the epidemiology and dynamics of BSE epidemics. Vet. Res., 39, 1-18.
Ducrot C., Sala C., Ru G., de Koeijer A. Sheridan H., Saegerman C., Selhorst T., Arnold M., Polak M.P., Calavas D., 2010. Modelling BSE trend over time in Europe, a risk assessment perspective. Eur. J. Epidemiol., 25, 411419.

Ducrot C., Paul M., Calavas D., 2013. BSE risk and the use of meat and bone meal in the feed industry: perspectives in the context of relaxing control measures. Natures Sci. Soc., 21, 1, 3-12.

Gajdusek D.C., 1977. Unconventional viruses and the Origin and Disappearance of Kuru. Science, 197, 943-960.

Hempel C., 1972. Eléments d'épistémologie. Armand Colin Editions, Paris, France, 184p.

Hill A.B., 1965. The environment and disease: Association or causation? Proc. Royal Soc. Med., 58, 295-300.

Hume D., édition 1983. Enquête sur l'entendement humain. Garnier Flammarion Editions, Paris, France, 247p.

Jarrige N., Ducrot C., Cazeau G., Morignat E., Calavas D., La Bonnardière C., 2007. Casecontrol study on feed risk factors for BSE cases born after the feed ban in France. Vet. Res., 38, 505-516.
Kant E., édition 1990. Critique de la raison pure. PUF Editions, Paris, France, 584p.

La Bonnardière C., Bonaiti B. Abrial D. Gasqui P., Calavas D., Ducrot C., Barnouin J., 2007. Milk yield, age at first calving, and the risk of BSE: An analysis at the farm level in France. Prev. Vet. Med., 78, 67-78.

Leibnitz G.W., édition 1969. Essais de Théodicée I., GF Editions, Paris, France, 502p.

Leibnitz G.W., édition 1990. Nouveaux essais sur l'entendement humain. GF Editions, Paris, France.

MacMahon B., Pugh T.F., 1970. Epidemiology: principles and methods. Little, Brown, Boston, 376p.

Popper K.R., 1973. La logique de la découverte scientifique. Payot Editions, Paris, France, 469p.

Russel B., édition 2002. La méthode scientifique en philosophie. Payot Editions, Paris, France, $304 \mathrm{p}$.

Wilesmith J.W., Wells G.A.H., Cranwell M.P., Ryan J.B.M., 1988. Bovine spongiform encephalopathy: epidemiological studies. Vet. Record, 123, 638-644.

Yakira E., 1993. La causalité de Galilée à Kant. PUF Editions, Paris, France, 128p.

\section{Résumé}

Différentes pratiques et méthodes, depuis la conception de l'étude jusqu'à l'analyse statistique, permettent à l'épidémiologiste de cerner au mieux la nature causale de la relation entre facteur et maladie, mais celle-ci relève de l'interprétation, car des circonstances non causales peuvent aussi induire une relation, le hasard mais aussi l'effet d'autres facteurs connectés. La philosophie identifie trois niveaux de discussion dans l'usage de la notion de causalité : le problème kantien de la distinction entre réalité absolue et réalité perçue par l'Homme, étant donné ses facultés de connaissance; le problème positiviste de la difficulté d'identifier des causes à partir de l'observation des phénomènes naturels, liaison entre phénomènes ne signifiant pas nécessairement succession causale ; le problème de l'induction, multiplier les expériences ne pouvant apporter de preuve définitive, les falsificationistes proposant d'étudier la robustesse d'un résultat en le soumettant à de nouveaux tests visant à le réfuter. Il ressort de cette réflexion interdisciplinaire différents apports à la pratique de l'épidémiologie et plus généralement de la recherche en biologie : entre autres rechercher les contextes les plus à même de remettre en question le résultat ; établir une conjonction de résultats différents et complémentaires pour conforter l'hypothèse de causalité, ce qui est en lien avec les dix critères de causalité utilisés par les épidémiologistes pour évaluer et discuter la nature causale d'une relation. En définitive, avoir un regard critique sévère sur les résultats d'étude est la seule façon d'apporter du crédit à l'interprétation quant à la causalité d'une relation statistique significative.

\section{Abstract}

\section{Philosophical perspective on causality in epidemiology - an interdisciplinary approach}

Different methods are used by the epidemiologist in order to get as close as possible to the causal nature of the relationship between a factor and a disease. However this is just an interpretation since different circumstances may randomly induce non-causal relationships between factors and disease, but also the effect of other interconnected factors. Philosophy identifies three levels of discussion in the use of causality: the distinction between absolute reality and the one perceived by man given his faculties of knowledge; the difficulty of identifying causes from the observation of natural phenomena because a link between phenomena does not necessarily mean causal succession; the problem of induction, in the sense that more conclusive experiments cannot provide definitive evidence, so that the falsificationists propose challenging the hypothesis by trying new tests to reject it. This interdisciplinary approach enhances the contribution of the philosophical perspective to the epidemiological practice, and more largely to research in biology : among others searching for study contexts adapted to challenge the results, establishing a combination of different and complementary arguments to get closer to causality, which draw a link to the ten causality criteria used by epidemiologists to evaluate and discuss the causal nature of a relationship. In conclusion, having a severe critical look at the result is the only way to add credit to the interpretation of a statistically significant relationship in terms of causality.

DUCROT C., BRES D., 2013. Regard philosophique sur la causalité en épidémiologie - approche interdisciplinaire. INRA Prod. Anim., 26, 4, 375-382. 